\title{
Stimulated secondary emission from semiconductor microcavities
}

Østergaard, John Erland; Mizeikis, V.; Langbein, Wolfgang Werner; Jensen, Jacob Riis; Hvam, Jørn Märcher

Published in:

Physical Review Letters

Link to article, DOI:

10.1103/PhysRevLett.86.5791

Publication date:

2001

Document Version

Publisher's PDF, also known as Version of record

Link back to DTU Orbit

Citation (APA):

Østergaard, J. E., Mizeikis, V., Langbein, W. W., Jensen, J. R., \& Hvam, J. M. (2001). Stimulated secondary emission from semiconductor microcavities. Physical Review Letters, 86(25), 5791-5794.

https://doi.org/10.1103/PhysRevLett.86.5791

\section{General rights}

Copyright and moral rights for the publications made accessible in the public portal are retained by the authors and/or other copyright owners and it is a condition of accessing publications that users recognise and abide by the legal requirements associated with these rights.

- Users may download and print one copy of any publication from the public portal for the purpose of private study or research.

- You may not further distribute the material or use it for any profit-making activity or commercial gain

- You may freely distribute the URL identifying the publication in the public portal

If you believe that this document breaches copyright please contact us providing details, and we will remove access to the work immediately and investigate your claim 


\title{
Stimulated Secondary Emission from Semiconductor Microcavities
}

\author{
J. Erland, V. Mizeikis, ${ }^{*}$ W. Langbein, ${ }^{\dagger}$ J. R. Jensen, and J. M. Hvam \\ Research Center COM, Technical University of Denmark, DK-2800 Kgs. Lyngby, Denmark
}

(Received 29 June 2000)

\begin{abstract}
We find strong influence of final-state stimulation on the time-resolved light emission dynamics from semiconductor microcavities after pulsed excitation allowing angle-resonant polariton-polariton scattering on the lower-polariton branch. The polariton dynamics can be controlled by injection of final-state polaritons at densities below a polariton saturation density of $5 \times 10^{8} \mathrm{~cm}^{-2}$. A bosonic enhancement factor in the dynamics of up to 700 is evaluated.
\end{abstract}

DOI: 10.1103/PhysRevLett.86.5791

Tailoring of light-matter interactions in semiconductor microcavities (MC) has been intensely studied since the first demonstration of strong coupling between the photon and exciton fields [1]. The new eigenstates, called cavity polaritons $(\mathrm{CP})$, have a strongly modified in-plane dispersion, as a function of in-plane wave vector $k_{\|}$, with a large Rabi splitting (several meV) expressing the anticrossing between the cavity photon dispersion and the quantum well exciton dispersion [2]. This modifies the coupling to light and consequently light emission processes from the MC. For instance, strong laserlike emission from $k_{\|} \sim 0$ polaritons is observed and attributed to bosonic enhancement in the emission process [3-5]. This relies on the bosonic nature of low-density polaritons leading to a stimulation of the scattering processes when the final $k$-state population is larger than one.

Recently, time-integrated pump-probe measurements have demonstrated stimulated polariton-polariton scattering (PPS) [6,7] as well as large parametric gain for a pulse injected at $k_{\|} \sim 0$ [8]. These stimulated processes are similar to those giving low-temperature lasing in II-VI compounds, first observed by Hvam [9], in the so-called "P"-band where stimulated PPS on the bulk polariton dispersion provides the gain. A number of concepts for bulk polariton lasing, such as the population inversion dynamics (see references in [9]), is analogous to the stimulated PPS in MC. The MC, though, provides photon localization in one dimension giving a nonzero density of states at $k_{\|}=0$ with a much longer polariton lifetime as a result. This enhances the gain process efficiently and provides very large gain coefficients in a very compact cavity [8]. It is thus interesting to consider this as a playground for new laser types, particularly in MC that may have polaritonic features preserved at room temperature. This possibility is relevant for polariton dampings smaller than a critical damping determined by the oscillator strength and the spatial dispersion coefficient in bulk systems [10]. The critical damping is yet unknown in MC systems, but a first estimate would be to compare to the Rabi splitting like in bulk.

The real-time dynamics of the emission is a key issue to understand the physics behind this prominent light emission process from a MC. A particular issue is how the
PACS numbers: 78.45. $+\mathrm{h}, 71.35 . \mathrm{Gg}, 78.47 .+\mathrm{p}$

real-time dynamics of the emission can be controlled by an external pulse using the final-state polariton population. In this Letter, we show strong evidence for final-state stimulation of angle-resonant PPS in time-resolved light emission experiments combining pulse-shaped ps pulses with streak camera time resolution of the MC emission. We observe a fast self-stimulated emission transient from the $k_{\|} \sim 0$ final-state distribution, after single-pulse excitation with large $k_{\|}$around a critical angle. This distribution is reflected in the angular spread of the emission and is correlated with the degree of quantum well disorder. The distribution of final states and thereby their populations are thus expected to be dependent on the inhomogeneous broadening. This is confirmed by transient measurements of the emission from homogeneous (HMC) as well as inhomogeneous (IMC) samples. In contrast to the excitation independent and slower dynamics in the IMC sample, the dynamics in the HMC sample is strongly density dependent with a shift of the emission maximum in time. The dynamics of $\mathrm{CP}$ is probed in more detail by seeding the emission at $k_{\|} \sim 0$ with a time-delayed pulse. This demonstrates a strong influence from stimulated scattering on the polariton dynamics on a time scale and with broadening dependences that can exclude freely decaying polarization effects as an explanation.

The experiments are made at a temperature of $5 \mathrm{~K}$ in a $\lambda$ cavity (bare linewidth of $\delta_{\mathrm{MC}}=260 \mu \mathrm{eV}$ ) with wedged thickness and a single homogeneously (inhomogeneously) broadened 25 (10) nm GaAs quantum well located at the antinode resulting in ultranarrow polariton linewidths of less than $200 \mu \mathrm{eV}$ [11-13]. The experimental configuration is shown in Fig. 1. In this work we concentrate on dynamics in the lower polariton (LP) branch at zero detuning where the polariton is $\sim 50 \%$ heavy-hole excitonlike and $\sim 50 \%$ photonlike. The polariton dispersions have previously been published [14]. The bare HMC quantum well has a homogeneous linewidth of $\delta_{\mathrm{HMC}}=120 \mu \mathrm{eV}<\delta_{\mathrm{MC}}$ minimizing disorder-related effects $[15,16]$, whereas the bare IMC quantum well has $\delta_{\mathrm{IMC}}=1 \mathrm{meV} \gg \delta_{\mathrm{MC}}$.

The dramatic change as a function of excitation power in the emission pattern from these $\mathrm{MC}$ is evident from the far-field images shown in Fig. 1. They are recorded at zero 


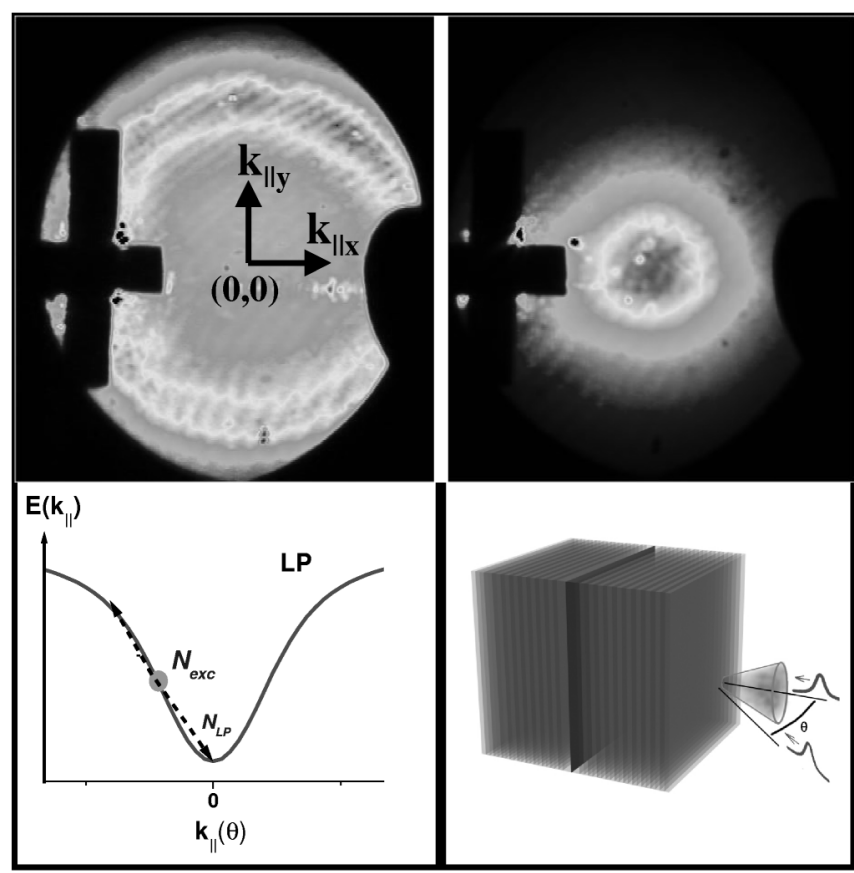

FIG. 1. The far-field emission change from a Rayleigh ring at low power $(\sim 50 \mu \mathrm{W}$, upper left) to strong emission close to $k \sim 0$ at higher power ( $>200 \mu \mathrm{W}$, upper right) following angle-resonant excitation in HMC. Notice the mirror and the block for the specular pump reflection. The excitation condition on the lower-polariton branch is sketched below to the left. Below to the right is shown the experimental configuration.

detuning and with single laser pulse excitation at a critical $k_{\|}(\theta)$, corresponding to an angle of $\theta \sim 12^{\circ}$. With low excitation density $\left(<10^{9} \mathrm{~cm}^{-2}\right)$, a ring distribution in $k$ space at $k_{\|}=1.4 \times 10^{4} \mathrm{~cm}^{-1}$ is observed due primarily to Rayleigh scattering of the incident light caused by residual inhomogeneous broadening [17]. At a higher excitation density, the emission pattern changes considerably to an intense narrow distribution around $k_{\|} \sim 0$. This emission depends strongly on the angle of excitation (within a few degrees) and on the detuning (within a few $\mathrm{meV}$ ), data not shown. The critical angle corresponds to the $k_{\|}$that is angle-resonant for PPS on the LP; see Fig. 1. Energy and wave vector are conserved in the angle-resonant process by scattering of two $k_{\|}=k_{\|, \text {,es }}$ polaritons into $2 k_{\|, \text {,es }}$ and $k_{\|} \sim 0$ states, respectively, the latter resulting in the strong emission. Before describing our time-resolved data, we note that the effect of the seeding on the $k_{\|}$-space resolved emission is very singular (data not shown). The selfstimulated emission has a full width half maximum in $k$ space corresponding to an external angle of $8^{\circ}\left(4^{\circ}\right)$ for the IMC (HMC) sample; however, only the emission from those $k_{\|}$states around $k_{\|} \sim 0$ excited by the seeding pulse is enhanced. This observation suggests that the mechanism of the transfer depends strongly on the occupation numbers, i.e., the number of polaritons per $k$ state, of the final $k_{\|}$states due to the bosonic nature of the polaritons.

In the following, we describe how the different occupation numbers in the HMC and IMC samples have pronounced influence on the dynamical response. We note that on average the occupation numbers are lower in the IMC sample simply because of the wider range of angleresonant $k$ states within the inhomogeneously broadened line $\left(\delta_{\text {IMC }} \gg \delta_{\text {MC }}\right)$. Figure 2 shows the time-resolved self-stimulated emissions from the two samples following single pulse excitation at $k_{\|, \text {,res }}$, which excludes coherent mixing with an externally induced polarization around $k_{\|} \sim 0$. The strong emission around $k_{\|} \sim 0$ is selected with a pinhole restricting the wave vector to a maximum of $k_{\|, \max }=3 \times 10^{3} \mathrm{~cm}^{-1}$. Three major differences between the two sets of real-time traces as a function of excitation density are seen as follows: (1) The self-stimulated transient is slower in the IMC sample relative to the HMC sample. (2) For increasing density, the emission maximum shifts to earlier time in the HMC sample in contrast to the IMC sample, where the emission maxima appear at the same time. (3) After the transient at time $>60 \mathrm{ps}$, the emission from the HMC is almost clamped to a fixed value for increasing density, whereas the emission time traces scale with the density in the IMC sample. These observations are consistent with the stronger influence from the

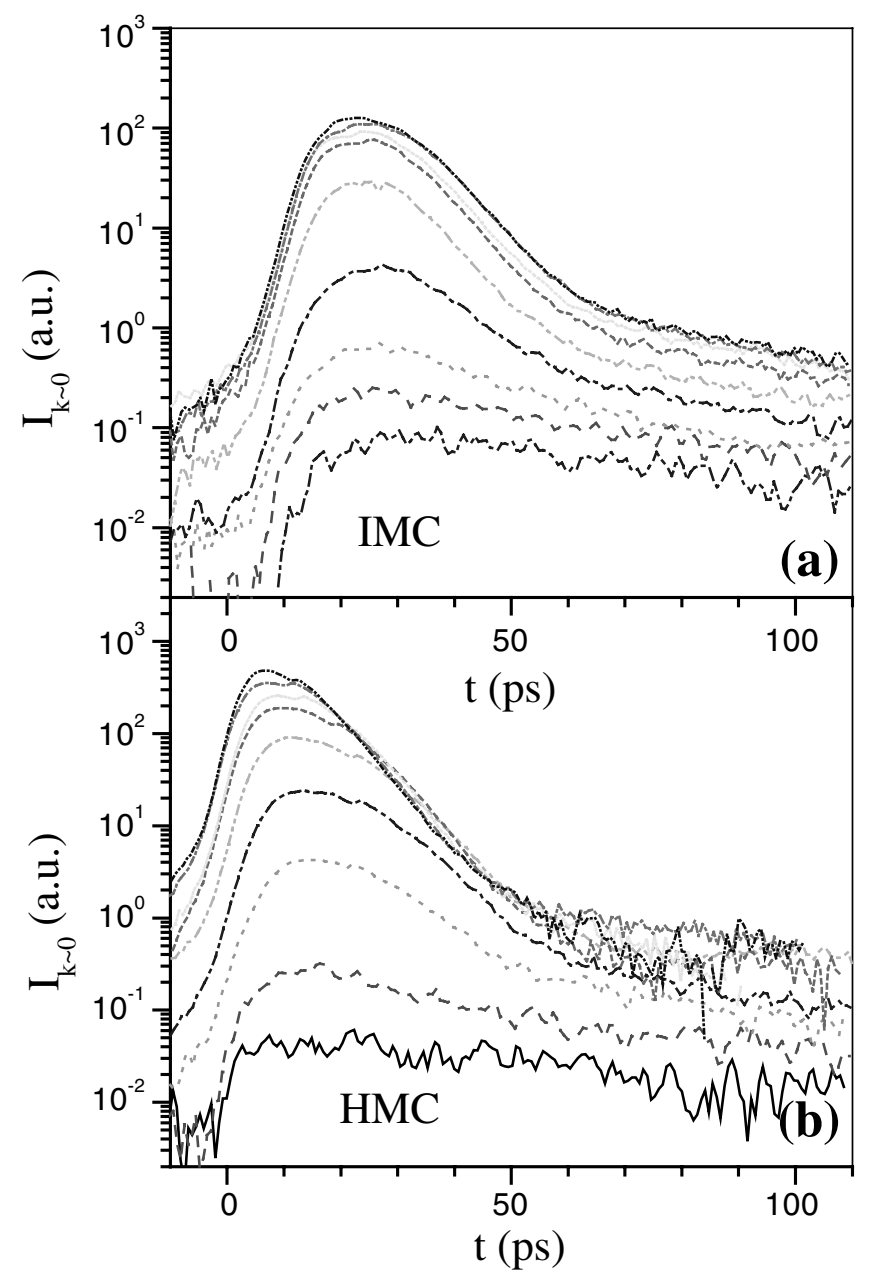

FIG. 2. Time-resolved emission for increasing excitation density $\left(n_{\mathrm{exc}}\right)$ for $(\mathrm{a})$ the IMC, with $n_{\mathrm{exc}}=(1.2,2.0,6.0,12,20$, $60,90,120,150) \times 10^{8} \mathrm{~cm}^{-2}$, and (b) the HMC, with $n_{\mathrm{exc}}=$ $(4.6,9.2,18,36,72,110,140,180,230) \times 10^{8} \mathrm{~cm}^{-2}$. 
higher occupation numbers in the HMC sample (for the same excitation density), enhancing the PPS rate. Our observations (2) and (3) suggest that the average occupation numbers in the IMC sample stay constant for stimulated PPS for increasing density, whereas the occupation number for the HMC sample continues to increase. Although wave mixing between different coherent polarizations in seeding experiments (see below) cannot be excluded, it cannot explain the data presented in Fig. 2 for the following reasons: (1) We inject only a coherent polariton population at the $k_{\| \text {,res }}$ states with zero laser-pulse overlap with the $k_{\|} \sim 0$ states. (2) The measured linewidth limits the influence of coherent wave mixing to a time scale of less than $10 \mathrm{ps,} \mathrm{much} \mathrm{shorter} \mathrm{than} \mathrm{the} \mathrm{transients} \mathrm{ob-}$ served in Fig. 2 [18]. (3) If freely decaying macroscopic polarizations should play a dominant role in the dynamical response, it should result in faster dynamics for increasing inhomogeneous broadening $\Gamma$, because of the well-known shortening of the dephasing time $\frac{1}{T_{2}^{*}}=\frac{1}{T_{2}}+\Gamma$, in contrast to what we observe [19]. We therefore conclude that there is a dominant contribution to the polariton dynamics in microcavities from population effects under angleresonant excitation conditions.

To probe the population dynamics further, we compare the self-stimulated emission to the one employing a seeding of the $k_{\|} \sim 0$ population with a second laser pulse delayed by 3 ps. The effect of the seeding pulse for both samples [Figs. 3(a) and 3(b)] is a shift of the emission maximum to earlier times and a strong enhancement of the

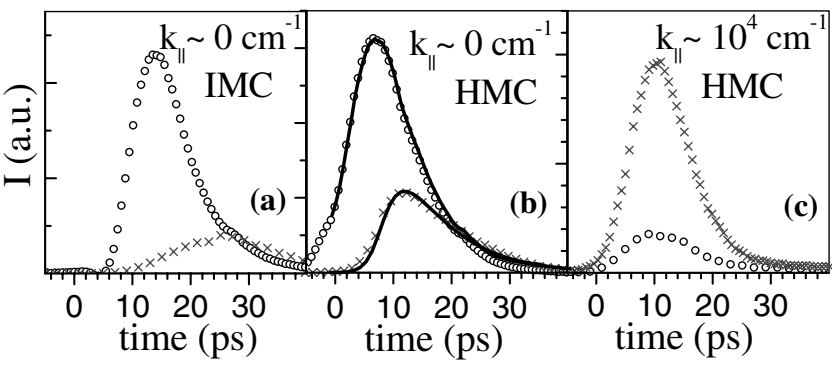

FIG. 3. The emission dynamics with (circles) and without (crosses) the seeding pulse detected at $k_{\|} \sim 0$ (a) for the IMC sample, (b) for the HMC sample, and at $k_{\|} \sim 10^{4} \mathrm{~cm}^{-1}$ (c) for the HMC sample. The full curves for HMC in (b) are simulations using a rate-equation model. The time $0 \mathrm{ps}$ corresponds to the arrival of the pump pulse.

emission. Note that the residual emission following the excitation with the seeding pulse, with the pump pulse closed, has been subtracted from all the time traces to probe the gain in the emission due to the seeding pulse directly. The effect of the seeding is strongest in the IMC sample where the main emission transient appears before the maximum self-stimulated emission would occur. This shows that the occupation number of the $k_{\|} \sim 0$ states, here increased by the seeding pulse, controls the emission dynamics.

To model this data we focus on the results from the HMC sample avoiding the complexity introduced by inhomogeneous broadening. The observed dynamical behavior is well described by simulations with coupled rate equations describing the PPS (fourth term) and polariton scattering with acoustic phonons (third term) as given in Ref. [7]:

$$
\begin{aligned}
& \frac{d \tilde{N}_{\mathrm{LP}}}{d t}=P_{\mathrm{LP}}(t)-\frac{\tilde{N}_{\mathrm{LP}}}{\tau_{\mathrm{LP}}}+a_{\mathrm{LP}, k=0} \frac{\tilde{N}_{\mathrm{exc}}}{S}\left(1+\tilde{N}_{\mathrm{LP}}\right)+\frac{1}{2} b_{\mathrm{LP}, k=0} \frac{\tilde{N}_{\mathrm{exc}}^{2}}{S^{2}}\left(1+\tilde{N}_{\mathrm{LP}}\right), \\
& \frac{d \tilde{N}_{\mathrm{exc}}}{d t}=P_{\mathrm{pump}}(t)-\frac{\tilde{N}_{\mathrm{exc}}}{\tau_{\mathrm{exc}}}-a_{\mathrm{LP}, k=0} \frac{\tilde{N}_{\mathrm{exc}}}{S}\left(1+\tilde{N}_{\mathrm{LP}}\right)-b_{\mathrm{LP}, k=0} \frac{\tilde{N}_{\mathrm{exc}}^{2}}{S^{2}}\left(1+\tilde{N}_{\mathrm{LP}}\right),
\end{aligned}
$$

where $\tilde{N}_{\mathrm{LP}}\left(\tilde{N}_{\text {exc }}\right)$ is the $k_{\|} \sim 0\left(k_{\|, \text {res }}\right)$ average polariton population per $k$ state, $P_{\mathrm{LP}}\left(P_{\text {pump }}\right)$ is the generation term from the seeding (pump) laser pulse, $\tau_{\mathrm{LP}}\left(\tau_{\mathrm{exc}}\right)$ is the polariton lifetime at $k_{\|} \sim 0\left(k_{\|, \text {res }}\right), a_{\mathrm{LP}, k=0}\left(b_{\mathrm{LP}, k=0}\right)$ is the coupling coefficient due to the phonon (polaritonpolariton) scattering and $S$ is the excitation area. The simulation based on Eqs. (1), shown in Fig. 3(b), perfectly matches the experiment with and without the seeding pulse using the measured lifetimes $\tau_{\mathrm{LP}}=7 \mathrm{ps}$ and $\tau_{\mathrm{exc}}=$ $100 \mathrm{ps}, a_{\mathrm{LP}, k=0} \sim 0$ and $b_{\mathrm{LP}, k=0}=2.4 \times 10^{-9} \mathrm{~cm}^{4} \mathrm{~s}^{-1}$. The PPS coefficient is comparable to the results of previous simulations [7]. We keep the angle-resonant conditions in this Letter such that the phonon scattering process is negligible compared to the strongly resonant PPS [3]. The self-stimulation of the PPS is caused by spontaneous buildup of the factor $\left(1+\tilde{N}_{\mathrm{LP}}\right)$ describing the bosonic enhancement of the scattering in Eqs. (1). From Eqs. (1) we evaluate a value of $\tilde{N}_{\mathrm{LP}}$ peaking at $\tilde{N}_{\mathrm{LP}, \max } \sim 700$ for the single-pulse data shown in Fig. 3(b). This value is consistent with a calculation of the polariton mode density given by $\rho=\int \frac{1}{(2 \pi)^{D}} d \mathbf{k}$. Integrating this over the pin- hole up to $k=k_{\|, \max }$ in $D=2$ dimensions gives $\rho=$ $7 \times 10^{5} \mathrm{~cm}^{-2}$. The resulting polariton density $\tilde{N}_{\mathrm{LP}, \max } \times$ $\rho=5 \times 10^{8} \mathrm{~cm}^{-2}$ is consistent with the estimate of the density used in the experiment. Although the model for the average population per $k$ state is rather simple, this evaluation demonstrates that, indeed, a high number of several hundred for the bosonic enhancement factor is obtained in this experiment as previously estimated in CW experiments [20]. The $\tilde{N}_{\mathrm{LP}}$ factor, controlling the stimulation process, can also be seeded as shown in Fig. 3. This demonstrates that a seeding pulse controls the emission process from a MC under resonant conditions. It also offers a controlled way of probing the dynamics because of the wellcharacterized polariton population created by the seeding pulse. We define a parametric gain factor $G_{\mathrm{BE}}$ as the ratio of the two time-integrated curves in Fig. 3(b) directly describing the gain in the emission due to the seeding pulse. For these data we get a value of $G_{\mathrm{BE}}$ up to 3 .

The consistency of our data is illustrated in Fig. 3(c), where we have selected the light emission at large $k_{\|}$close 


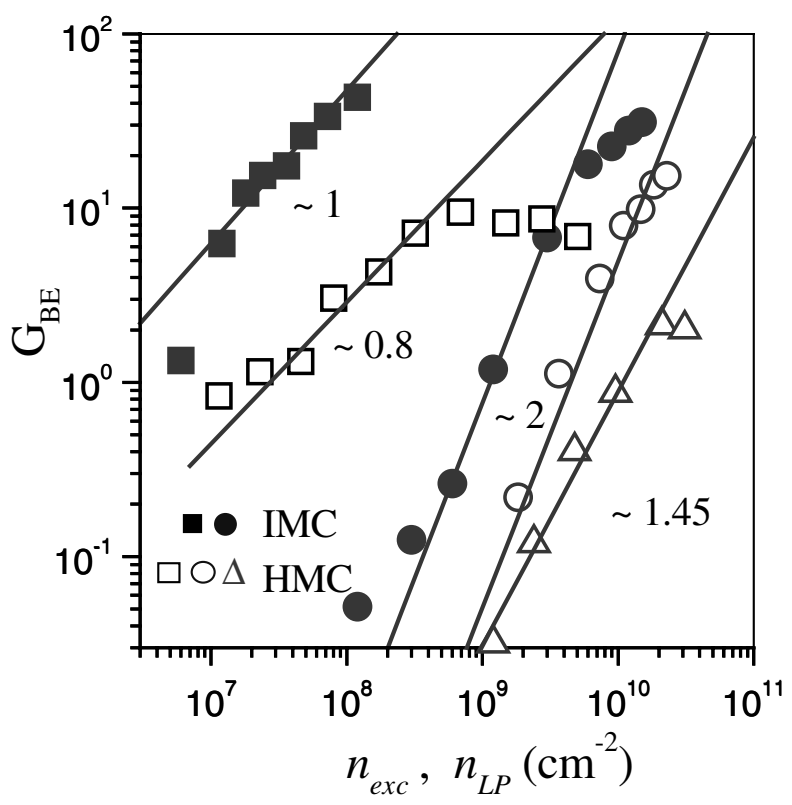

FIG. 4. Parametric gain factor $G_{\mathrm{BE}}$ of the $k_{\|} \sim 0$ emission relative to the spontaneous emission for the self-stimulation (circles) as a function of the excited polariton density $n_{\text {exc }}$ and due to the seeding (squares) as a function of the injected polariton density $n_{\mathrm{LP}}$ at $k_{\|} \sim 0$ (with $n_{\mathrm{exc}, \mathrm{HMC}}=2.3 \times 10^{10} \mathrm{~cm}^{-2}$ and $n_{\text {exc,IMC }}=1.6 \times 10^{9} \mathrm{~cm}^{-2}$ ) for the IMC sample (closed symbols) and the HMC sample (open symbols). The enhancement of the emission from $k_{\|} \sim 10^{4} \mathrm{~cm}^{-1}$ as a function of density $n_{\mathrm{exc}}$ is shown for the HMC sample demonstrating stimulated removal (open triangles).

to the excitation but otherwise kept the conditions similar to those in Fig. 3(a). In this case, we observe a negative $G_{\mathrm{BE}}$ as a result of the stimulated removal of polaritons due to the seeding pulse leading to a much smaller polariton population for the subsequent light emission at that $k_{\|}$ value. We can therefore consistently probe the effect of the seeding pulse and the stimulated processes not only in the final state but also in the initial state.

The dynamical behavior of the stimulated PPS is discussed in the following from experiments probing the variation of the $G_{\mathrm{BE}}$ with excitation densities summarized in Fig. 4. The excitation density dependence of the $G_{\mathrm{BE}}$ is predicted from Eqs. (1) to be near linear (quadratic) in the injected polariton populations $n_{\mathrm{LP}} S\left(n_{\mathrm{exc}} S\right)$, which is consistent with our observations shown in Fig. 4. The deviations from these dependencies at higher excitation densities correspond to a breakdown of the bosonic condition for the polariton stimulation. The excitonic saturation density in a quantum well $n_{\text {exc }}=4 \times 10^{10} \mathrm{~cm}^{-2}$ matches the turnover of the self-stimulated emission [21]. The lower saturation density $\sim 5 \times 10^{8} \mathrm{~cm}^{-2}$ for the $n_{\mathrm{LP}}$ data is almost 2 orders of magnitude smaller providing a direct measure of the $k_{\|} \sim 0$ polariton density for saturation of the bosonic enhancement. This density is much lower than the calculated densities $>10^{10} \mathrm{~cm}^{-2}$ for which the Bose commutator is less than 1 [5]. From this we can conclude that our measurements are performed in the low-density regime where the bosonic character of the polaritons is preserved.

From the data in Fig. 4 we notice that the $G_{\mathrm{BE}}$ is much larger for the IMC sample compared to the HMC sample for optimized conditions. The largest $G_{\mathrm{BE}}$ found for the HMC sample was close to 10 , while the IMC sample showed an enhancement as large as 40 . This has previously been attributed to relaxation involving localized states in IMC samples that is absent in our HMC sample [22].

In conclusion, we found a significant influence on emission dynamics in homogeneously broadened microcavities from controlling the average occupation numbers of finite-mass polariton states involved in stimulation processes on the LP. These bosonic enhancement effects have demonstrated a richness of light-matter interactions in MC that gives new possibilities for fundamental studies in physics and applications to new optical devices.

Technical assistance from K. Leosson and N.A. Mortensen, and discussions with D. Birkedal are gratefully acknowledged. V. M. acknowledges financial support from Danish Rectors Conference. The MC samples were grown in III-V Nanolab. This work was supported by the Danish Natural Sciences Research Council.

*Permanent address: Institute of Materials Research and Applied Science, Vilnius University, LT-2000 Vilnius, Lithuania.

†Permanent address: Lehrstuhl für Experimentelle Physik EIIb, Universität Dortmund, D-44221 Dortmund, Germany.

[1] C. Weisbuch et al., Phys. Rev. Lett. 69, 3314 (1992).

[2] R. Houdré et al., Phys. Rev. Lett. 73, 2043 (1994).

[3] P. Senellart et al., Phys. Rev. Lett. 82, 1233 (1999).

[4] Le Si Dang et al., Phys. Rev. Lett. 81, 3920 (1998).

[5] M. Kira et al., Phys. Rev. Lett. 79, 5170 (1997).

[6] R. Huang et al., Phys. Rev. B 61, R7854 (2000).

[7] F. Tassone et al., Phys. Rev. B 59, 10830 (1999).

[8] P. G. Savvidis et al., Phys. Rev. Lett. 84, 1547 (2000).

[9] J. M. Hvam, Solid State Commun. 12, 95 (1973).

[10] A. C. Schaefer et al., Phys. Rev. Lett. 79, 4870 (1997).

[11] J. R. Jensen et al., Appl. Phys. Lett. 76, 3262 (2000).

[12] W. Langbein et al., Phys. Rev. B 61, 1692 (2000).

[13] P. Borri et al., Phys. Rev. B 63, 35307 (2001).

[14] J. Erland et al., Phys. Status Solidi (b) 221, 115 (2000).

[15] D. M. Whittaker, Phys. Rev. Lett. 80, 4791 (1998).

[16] C. Ell et al., Phys. Rev. Lett. 80, 4795 (1998).

[17] A. V. Shchegrov et al., Phys. Rev. Lett. 84, 3478 (2000).

[18] Y.-S. Lee et al., Phys. Rev. Lett. 83, 5338 (1999); T. Baars et al., Phys. Rev. B 61, R2409 (2000).

[19] L. Allen and J. H. Eberly, Optical Resonance and Two-level Atoms (Dover, New York, 1987); J. Erland et al., Phys. Rev. B 50, 15047 (1994).

[20] R. M. Stevenson et al., Phys. Rev. Lett. 85, 3680 (2000).

[21] S. Schmitt-Rink et al., Adv. Phys. 38, 89 (1989).

[22] V.D. Kulakovskii et al., in Proccedings of the COST268 Workshop, Wurzburg, Germany, 2001 (to be published). 